Enfoques. Revista de Investigación en Ciencias de la Administración http://doi.org/10.33996/revistaenfoques.v3i9.53

No. 9 | Volumen 3 | Enero - Marzo 2019

\section{EMPRENDIMIENTO Y RESILIENCIA: MITOS Y REALIDADES REFERENTE: ENTIDAD NO GUBERNAMENTAL EN EL ÁREA DEL EMPRENDIMIENTO}

\author{
ENTREPRENEURSHIP AND RESILIENCE: MYTHS AND REALITIES \\ CONCERNING: NON-GOVERNMENTAL ENTITY IN THE AREA OF \\ ENTREPRENEURSHIP
}

Laurymar Pérez, Johana Pérez y Dana Parada
Artículo recibido octubre 2018 | Arbitrado noviembre 2018 | Publicado 01 de enero 2019

\section{Resumen}

El artículo que se muestra a continuación buscó analizar el emprendimiento y la resiliencia desde la perspectiva de los mitos y realidades en una Entidad no gubernamental en el área del emprendimiento. Las teorías utilizadas fueron la Teoría del Mercado Laboral de Schumpeter la Teoría de la Segmentación de los Mercados. Así como la Teoría del riesgo y trabajo. EI método de investigación que se desarrolló en el área del emprendimiento se enfocó desde la perspectiva del pensamiento meta-positivo, siendo un estudio en la modalidad etnográfica en el paradigma cualitativo, utilizando técnicas e instrumentos cualitativos. Los informantes claves fueron tres estudiantes, tres egresados y tres expertos a los cuales se les aplicaron la entrevista. A partir de los hallazgos encontrados que la diferencia entre el emprendedor y el individuo común la establece su actitud. El emprendedor es una persona con capacidad de crear, de llevar adelante sus ideas, de generar bienes y servicios, de asumir riesgos y de enfrentar problemas.

Palabras clave: emprendimiento; resiliencia; mitos; realidades

\begin{abstract}
The article shown below sought to analyze entrepreneurship and resilience from the perspective of myths and realities in a non-governmental entity in the area of entrepreneurship. The theories used were the Theory of the Labor Market of Schumpeter the Theory of Market Segmentation. As well as the Theory of risk and work. The research method that was developed in the area of entrepreneurship was focused from the perspective of metapositive thinking, being a study in the ethnographic modality in the qualitative paradigm, using qualitative techniques and instruments. The key informants were three students, three graduates and three experts to whom the interview was applied. From the findings found that the difference between the entrepreneur and the common individual is established by his attitude. The entrepreneur is a person with the capacity to create, to carry out his ideas, to generate goods and services, to take risks and to face problems.
\end{abstract}

Palabras clave: emprendimiento; resiliencia; mitos; realidades

\section{Laurymar Pérez \\ lauriizp@gmail.com \\ Universidad de Carabobo, Venezuela \\ Licenciada en Relaciones Industriales Universidad de Carabobo, Venezuela. Sindicalismo y Relaciones Laborales (FUNDAJOSVAR) Seguridad Social E Industrial (CLRI). \\ Johana Pérez \\ jcpr2006@hotmail.com \\ Universidad de Carabobo, Venezuela \\ Licenciada en Relaciones Industriales Universidad de Carabobo. \\ Dana Parada \\ dari_1551@hotmail.com Universidad de Carabobo, Venezuela \\ Licenciada en Relaciones Industriales Universidad de Carabobo, Venezuela. Especialista en Sistema de Gestión por Competencias Nuevos Paradigmas de RRHH Cooperativismo.}




\section{INTRODUCCIÓN}

Desde hace ya varios años se ha ido observando que por situaciones de diversa índole, las personas y las organizaciones del país se han visto en la necesidad de poner a prueba la capacidad de reacción para adaptarse a las dificultades y continuar su actividad del día a día, con mayor éxito, para no verse afectadas por sucesos de diferente naturaleza y dimensión, lo cual ha generado que poco a poco las acciones y actitudes emprendedoras y resiliente estén en constante proceso de evolución, con resultados cada vez más satisfactorios.

La investigación centra su interés en el estudio del emprendimiento y la resiliencia desde la perspectiva laboral o procesos de trabajo donde se puede observar como la cualidad, experticias, competencias que tienen las personas dentro de las organizaciones para emprender nuevos proyectos, y la manera como ponen en práctica sus capacidades, potencialidades y fortalezas, para desarrollar el proceso de afrontar, los mitos y realidades transformándolos y convirtiéndolos en una oportunidad para enfrentarlos, luchar $y$ vencer.

Se puede deducir la relación íntima que existe entre estos dos procesos y el ciclo vital de las personas y las organizaciones donde se establecen las relaciones de trabajo ya que en todas las etapas están condicionadas a realizar ajustes $y$ medidas en sus ideologías, conductas, credos y relaciones, con el objetivo de lograr una mejor y más placentera adaptación consigo mismo y con su entorno laboral, familiar, social, cultural entre otros.

Para algunos investigadores, la resiliencia es las dos caras de una misma moneda, ya que es la capacidad de enfrentar las dificultades y salir fortalecidos de las mismas, el emprendimiento es considerado hoy en día como factor propio dentro de la competitividad y liderazgo, involucra respuestas que pueden darse tanto de forma individual como colectiva. Las personas se asumen como parte del grupo y reconocen que necesitan de él para su desarrollo en una relación de mutua influencia.

En la actualidad se han estado observando procesos de cambio y profundas crisis económicas a nivel mundial. Existen personas, que a pesar de vivir en condiciones de dificultad, son capaces de desarrollar conductas que les facilitan tener una buena calidad de vida. Para ello se toma como referencia en esta investigación el concepto de Resiliencia, propuesto por Werner (2005:56) quien dice que la resiliencia "es un concepto tomado y desarrollado por la psicología evolutiva y social y es definida como la capacidad que tiene el individuo o ciertos grupos de enfrenar situaciones traumáticas y de estrés y sobreponerse de manera exitosa a ellas". También es importante destacar que Melillo Suárez (2001) quien afirma que:

La resiliencia se produce en función de procesos sociales intra psíquicos, no se nace resiliente ni se adquiere naturalmente en el desarrollo. Si no que esta depende de interacciones del sujeto con otros seres humanos responsables de la construcción del sistema psíquicos (p. 87).

El uso del concepto resiliencia es reciente en el campo organizacional, quizá por su origen teórico. Dentro de este campo disciplinario se le emplea como una forma de designar la capacidad de recuperación de las organizaciones ante un evento no esperado, también se le puede considerar como un detonante de acciones de previsión o como parte toral de un proceso estratégico. 
La resiliencia en la organización puede ser definida como la capacidad de un sistema para absorber los cambios, que se vislumbran como una serie de crisis repentinas (López, 2009; Smith y Graetz, 2011), y aún conservar su funcionalidad esencial.

En las relaciones de trabajo la resiliencia puede ser vista como la capacidad para evitar las consecuencias negativas de un acontecimiento ya producido; también, como la capacidad de prevenir alguna situación con consecuencias negativas agudizadas al transcurrir el tiempo $\mathrm{y}$, por último, se asume como la capacidad de recuperación después de haber hecho frente a las consecuencias negativas de un evento (Westrum, 2006:7).

La resiliencia expresa la capacidad de una organización $\mathrm{o}$ de sus partes para responder rápidamente a la incertidumbre. En consecuencia, la resiliencia es un estado de la organización producido por la administración de la incertidumbre dentro de un sistema adaptativo complejo. Un indicador de este estado se encuentra en una organización con una ingente capacidad adaptativa (Dahms, 2010:22). La resiliencia no es una condición estática de una organización, puede variar con el tiempo en función de la naturaleza y las consecuencias de una crisis en particular.

La resiliencia de una organización probablemente se encuentra relacionada de manera directa o indirecta con la resiliencia de otras organizaciones (clientes, proveedores, agentes gubernamentales e, incluso, competidores). Adicionalmente, una organización depende de la resiliencia individual de su personal y de las comunidades ligadas a ella en una relación simbiótica. Como resultado de esta interacción de doble vía una organización a su vez participa en el fortalecimiento de las capacidades de resistencia de dichas comunidades.

Por su parte, el emprendimiento es un término últimamente muy utilizado en todo el mundo. Aunque el emprendimiento siempre ha estado presente a lo largo de la historia de la humanidad, pues es inherente a ésta, en las últimas décadas, éste concepto se ha vuelto de suma importancia, ante la necesidad de superar los constantes y crecientes problemas económicos.

Para Torres (2014):

La palabra emprendimiento
proviene del francés
entrepreneur (pionero), y se
refiere a la capacidad de una
persona para hacer un esfuerzo
adicional por alcanzar una meta
u objetivo, siendo utilizada
también para referirse a la
persona que iniciaba una nueva
empresa o proyecto, término
que después fue aplicado a
empresarios que fueron
innovadores o agregaban valor a
un producto o proceso ya
existente (p. 6).

Según lo expresado por el autor, el emprendimiento es aquella actitud y aptitud de la persona que le permite emprender nuevos retos, nuevos proyectos; es lo que le permite avanzar un paso más, ir más allá de donde ya ha llegado. Es lo que hace que una persona esté insatisfecha con lo que es y lo que ha logrado, y como consecuencia de ello quiera alcanzar mayores logros.

Normalmente se asocia la figura del emprendedor con personas con espíritu innovador $y$ adverso al riesgo en donde percibe una oportunidad y crea una organización para perseguirla, perfil que hasta cierto grado tiene hasta carácter épico. Pero también es cierto que el 
emprendimiento se puede atribuir a las organizaciones, en especial a las empresas que logran crear entornos favorables a la iniciativa emprendedora, sin embargo aún se encuentran organizaciones que no permiten la iniciativa de sus miembros, ya sea por burocratización o miedo al cambio. Implementar un modelo que ayude la iniciativa emprendedora requiere un cambio de mentalidad.

En el ambiente competitivo de hoy, según Melillo y Suárez (2001) las empresas deben permitir los espacios para que incorporen nuevas líneas de productos, innovar en los servicios existentes, entre otros, es por eso que para que una organización tenga características de emprendedora deben poseer una estructura que permita desarrollar la explotación y la exploración creativa, poseer directivos que vean su misión como una posibilidad de creación que desarrolle el talento emprendedor y que entiendan el concepto de emprender desde el interior de la organización.

Una organización para que sea emprendedora, deberá permitir y facilitar que las personas que la componen, se comporten dando facilidades para la iniciativa dentro de la organización, estimulando nuevas oportunidades en la organización, entregando recursos y abiertos a los cambios. El desarrollo del espíritu emprendedor al interior de una organización, puede permitir desarrollar nuevos proyectos o también renovar con nuevas ideas.

Hasta el momento, muchos han sido los autores que han hablado acerca de estos temas emprendimiento y resiliencia organizacional, reflejando que la crisis ha cambiado a las personas, tomando en cuenta que la resiliencia no es algo común que se presenta automáticamente en el ser humano
- en las organizaciones donde se desempeña, para tenerla requiere que la persona esté convencida de que si puede salir adelante. Cabe destacar que cada individuo $\mathrm{u}$ organización en el contexto de las relaciones de trabajo reacciona diferente frente a las situaciones que se les presentan, algunos tienen actitudes positivas, tomando la iniciativa de llevar adelante un proyecto superando los obstáculos y tratando de sacar aprendizajes de estas situaciones y otros se quedan estancados en sus dificultades.

Es por ello, que el estado Carabobo, en el cual existía un gran número de organizaciones empresariales, las cuales se han visto afectadas económicamente por la política cambiaria que atraviesa el país en la actualidad lo cual ha ido agudizando el problema, motivo por el cual las ciudadanos se han visto en la necesidad de desarrollar el emprendimiento y la resiliencia personal que no es más que la actitud que asumen las personas que le permite iniciar nuevos retos, proyectos, entre otros, utilizándolas como un medio de supervivencia, por la insatisfacción que se produce al no poder cubrir sus necesidades y como consecuencia de ello, utilizan estos medios para alcanzar mayores logros en sus vidas.

En el caso específico de la Asociación Civil Juventud y Trabajo que se encarga de capacitar a jóvenes para que sean insertados en el campo laboral. Dicha Asociación Civil se encuentra afiliada a la Asociación Civil venezolana de Educación Católica perteneciente al Ministerio de Educación, Cultura y Deportes. Actualmente atienden 500 estudiantes en todo el Estado Carabobo con edades comprendidas entre 16 y 30 años. Allí reciben cursos de electricidad, refrigeración, asistente administrativo, máquinas y herramientas. Los instructores son los encargados de preparar profesionalmente a los estudiantes y el más 
sobresaliente tiene la oportunidad de ser colocado en el Parque Industrial como candidato potencial a un puesto de trabajo. También bajo alianza del INCE se consigue que se les pague a los estudiantes un sueldo mientras aprenden para así lograr el emprendimiento.

Para el estudio del emprendimiento y la resiliencia mitos y realidades fueron tomados las siguientes interrogantes la cual dieron orientación al estudio que analiza en este artículo ¿Qué cualidades tienen las personas emprendedoras?, ¿Cuáles son los mitos y realidades que existen en el entorno de las personas emprendedoras?, ¿Cuáles son los mitos y realidades que existen en el entorno de las personas resilientes? y ¿Qué actitudes tienen las personas resilientes?

De acuerdo a las interrogantes que se propuestos en este estudio surgieron los siguientes objetivos: Analizar el emprendimiento y la resiliencia desde la perspectiva de los mitos y realidades en una Entidad no gubernamental en el área del emprendimiento. También el emprendimiento desde la perspectiva del trabajo en una sociedad de riesgo y los factores socio económicos que permiten evaluar la existencia de betas de emprendimiento asociada a la búsqueda de inserción con conductas resilientes; además de analizar las representaciones sociales de los emprendedores resilientes en términos de mitos y realidades; a fin de determinar las opiniones que tienen las personas resilientes en situaciones del emprendimiento.

Para finalizar se tiene que el propósito fue constatar la realidad objetiva sobre el tema y conocer por que las personas emprendedoras y resiliente en un momento de crisis tienen la capacidad para emprender nuevos objetivos. Ello contribuye al incremento del conocimiento, ya que por medio de la investigación se busca, aprender y obtener nuevas ideas que aporten beneficios al aprendizaje.

La investigación constituye un antecedente importante para futuras investigaciones por cuanto podrá ser usada como literatura para las personas interesadas en conocer sobre emprendimiento la resiliencia mitos y realidades en una organización no gubernamental en Valencia estado, Carabobo, con la finalidad de cambiar la forma en que las personas interpretan cada circunstancia. Hoy en día existe la tendencia de emprender desde el punto de vista de la resiliencia, a partir de las potencialidades de la persona en relación con su entorno. Por eso es importante distinguir los mitos y realidades que se presentan, y sobre todo tomar acciones que faciliten un proceso adecuado para su desarrollo.

\section{MÉTODO}

El estudio se llevó a cabo mediante el paradigma cualitativo, el método de investigación que se desarrolló para la interpretación del emprendimiento y la resiliencia desde la perspectiva de los mitos y realidades en una entidad no gubernamental en el área del emprendimiento, desde la perspectiva del pensamiento meta-positivo bajo la modalidad etnográfica, en la cual el Ethnos de esta investigación se constituye dentro del aula de clase, y sus diversas manifestaciones en relación al proceso de convivencia. Para efecto de esta investigación se tomó como escenario la Asociación Civil Juventud y Trabajo a fin de llevar a cabo la investigación etnográfica.

Contó con una población perteneciente a la Asociación Civil Juventud y Trabajo que se encarga de capacitar a jóvenes para que sean insertados en el campo laboral. 
Dicha Asociación Civil se encuentra afiliada a la Asociación Civil venezolana de Educación Católica perteneciente al Ministerio de Educación, Cultura y Deportes. Actualmente atienden 500 estudiantes en todo el estado Carabobo con edades comprendidas entre 16 y 30 años. Allí reciben cursos de electricidad, refrigeración, asistente administrativo, máquinas y herramientas. Así como expertos de la Universidad de Carabobo.

\section{RESULTADOS Y DISCUSIÓN}

A continuación en este apartado se muestran algunos aportes significativos a los efectos de los análisis e interpretación de los hallazgos del estudio

\section{Sistema de categorías claves}

Con relación las categorías principales se destacan: autodefinición de emprendedor, características del emprendedor, auto reconocimiento del "yo" emprendedor, origen de la actitud emprendedora, clave para el triunfo profesional del emprendedor, elementos que contribuyen al emprendimiento, capacitación y emprendimiento, auto significado de resiliencia, autoreconocimiento como persona resiliente y la conducta resiliente como mito o realidad.

\section{Los sujetos y su visión del problema}

\section{Los sujetos ponen orden discursivo: criterios y contenidos}

Este sistema de categorías surgió de las entrevistas realizadas a tres (3) estudiantes, tres (3) expertos y tres (3) egresados, donde se pudo evidenciar a manera general que la persona emprendedora es aquella que se cree capaz de iniciar su propia empresa, de tomar sus propias decisiones, tiene ideas, conocimientos de lo quiere lograr, sin importar el riesgo que se pueda presentar en el desarrollo de sus actividades. Además debe tener iniciativa y buscar su propio destino laboral creyendo en sus ideales, caracterizándose por ser valiente y capaz. Entendiéndose que el emprendimiento, es entonces la posibilidad que tienen las personas que han estudiado la necesidad de un producto, proceso, un bien para uso colectivo entre otros, para proceder a fabricarlos, a negociarlo organizándose e invirtiendo dinero, el cual implica correr riesgos, esto con la finalidad de crear un beneficio en la comunidad para solucionar una necesidad en la misma.

El reconocerse como emprendedor es reconocer que se es una persona que habitualmente siente que está sometida a muchísimas evaluaciones: la suya propia, la de sus socios, equipo de trabajo, inversores, proveedores... y que, a la vez, evalúa al resto. Por eso esto les parece especialmente interesante, a la par que contribuirá a su propio desarrollo y al de su negocio, el saber dar y recibir reconocimiento. Además citan la motivación del empresario como principal motivo para aprovechar la oportunidad de emprender. Se plantean diversas razones: motivación primaria de emprendimiento o deseo de poner un negocio, existencia de oportunidades, modelo de referencia o haber conocido personalmente a un emprendedor en los dos últimos años, autoempleo, expectativas de ingresos, y miedo al fracaso para emprender, es decir, si en el país o región la mayoría de la gente opina que iniciar un negocio es una opción profesional atractiva.

El emprendedor se mueve con libertad, es por ello, que siempre presenta la perseverancia y la determinación como experiencias frecuentes que reflejan su confianza y entusiasmo por la novedad. Lo cierto es que en esto del emprendimiento 
existen muchos mitos y realidades se dice que para ser emprendedor se debe nacer con esa cualidad y no tratar de serlo en el camino, pero en realidad se puede tener inteligencia innata, llevando a convertir a las personas en emprendedores por acumulación de habilidades, conocimiento práctico, experiencia y contactos.

A esto se suma que los entrevistados opinen que existen muchas definiciones de Resiliencia aunque todas tienen siempre un común denominador, que no es más que la capacidad de una persona de hacer las cosas bien pese a las condiciones que haya tenido en su vida, superarlas frustraciones y salir de ellas reforzado o incluso renovado, recuperándose y permitiéndose tener una vida significativa y productiva para sí y para la sociedad en la que se encuentra inserto. Pero la resiliencia no es una condición o una cualidad innata, algo que tenemos o no tenemos en función de los caprichos del destino o de nuestra buena fortuna. Es algo que todos, sin excepción, podemos desarrollar, aunque en ocasiones para lograrlo sea necesario modificar algunas de nuestras creencias, convicciones y hábitos.

\section{Análisis las debilidades y fortalezas}

Con respecto a las fortalezas de las categorías extraídas, bien pudiera decirse que a pesar de las diferencias que pudieron tener los encuestados en cuanto a la información que manejan desde su perspectiva de estudiantes, egresados 0 expertos, todos tienen una idea a manera general de lo que significa el emprendimiento y la resiliencia, no presentando dificultades a la hora de definir estos términos. Las debilidades estarían en todo caso al momento de establecer los factores que pudieran contribuir $u$ obstaculizar el emprendimiento, ya que algunos destacan el factor económico como preponderante y los expertos el aspecto personal.

Teorización triangular: construcción de la visión en su conjunto

Al triangular las categorías desde la visión de los entrevistados existieron elementos comunes en cuanto a la autodefinición de emprendedor, características del emprendedor, auto reconocimiento del "yo" emprendedor, clave para el triunfo profesional del emprendedor, elementos que contribuyen al emprendimiento, capacitación y emprendimiento, auto significado de resiliencia, autoreconocimiento como persona resiliente y la conducta resiliente como mito o realidad. (Ver cuadro 1). 
Cuadro 1. Categorías extraídas de las entrevistas realizadas

\begin{tabular}{|c|c|c|}
\hline $\begin{array}{l}\text { CATEGORÍAS EXTRAÍDAS DE } \\
\text { LOS ESTUDIANTES }\end{array}$ & $\begin{array}{l}\text { CATEGORÍAS EXTRAIIDAS } \\
\text { DE LOS EGRESADOS }\end{array}$ & $\begin{array}{l}\text { CATEGORÍAS EXTRAIIDAS } \\
\text { DE LOS EXPERTOS }\end{array}$ \\
\hline $\begin{array}{l}\text { Autodefinición del } \\
\text { emprendedor }\end{array}$ & $\begin{array}{l}\text { Autodefinición del } \\
\text { emprendedor }\end{array}$ & $\begin{array}{l}\text { Autodefinición del } \\
\text { emprendedor }\end{array}$ \\
\hline $\begin{array}{l}\text { Características del } \\
\text { Emprendedor }\end{array}$ & $\begin{array}{l}\text { Factores que contribuyen al } \\
\text { emprendimiento }\end{array}$ & $\begin{array}{l}\text { Características del } \\
\text { Emprendedor en términos } \\
\text { generales }\end{array}$ \\
\hline $\begin{array}{l}\text { Auto reconocimiento del "yo" } \\
\text { emprendedor }\end{array}$ & $\begin{array}{l}\text { Clave para el triunfo } \\
\text { profesional del emprendedor }\end{array}$ & Capacitación y emprendimiento \\
\hline $\begin{array}{l}\text { Origen de la actitud } \\
\text { emprendedora }\end{array}$ & $\begin{array}{l}\text { Auto reconocimiento de } \\
\text { aptitudes emprendedoras }\end{array}$ & $\begin{array}{l}\text { Origen de la actitud } \\
\text { emprendedora }\end{array}$ \\
\hline $\begin{array}{l}\text { Elementos que contribuyen al } \\
\text { emprendimiento }\end{array}$ & $\begin{array}{l}\text { Limitantes del } \\
\text { emprendimiento }\end{array}$ & $\begin{array}{l}\text { Clarificar la conducta resiliente } \\
\text { como mito o realidad }\end{array}$ \\
\hline $\begin{array}{l}\text { Capacitación y } \\
\text { emprendimiento }\end{array}$ & Auto significado de resiliencia & $\begin{array}{l}\text { Resistencia al cambio y } \\
\text { resiliencia }\end{array}$ \\
\hline Auto significado de resiliencia & $\begin{array}{l}\text { Autor reconocimiento como } \\
\text { persona resiliente }\end{array}$ & $\begin{array}{l}\text { Diferencias entre } \\
\text { emprendimiento y Resiliencia }\end{array}$ \\
\hline $\begin{array}{l}\text { Autoreconocimiento como } \\
\text { persona resiliente }\end{array}$ & $\begin{array}{l}\text { Caracterizar a la persona } \\
\text { resiliente }\end{array}$ & $\begin{array}{l}\text { Emprendimiento como mito o } \\
\text { realidad }\end{array}$ \\
\hline $\begin{array}{l}\text { Elementos favorecedores de la } \\
\text { resiliencia }\end{array}$ & $\begin{array}{l}\text { Clarificar la conducta resiliente } \\
\text { como mito o realidad }\end{array}$ & \\
\hline $\begin{array}{l}\text { Factores obstaculizantes de la } \\
\text { resiliencia }\end{array}$ & & \\
\hline
\end{tabular}

Fuente: Pérez, Pérez y Parada (2017)

Tal como pudo observar en el cuadro 1 se puede finalizar que existen diversas categorías para analizar el emprendimiento y la resiliencia desde la perspectiva de los mitos y realidades en una Entidad no gubernamental en el área del emprendimiento. Permitiendo una visión global a través de los entrevistados quienes expresaron su opinión acerca de ambas definiciones, unos de manera empírica y otros como los expertos con mayor conocimiento del tema y utilizando un lenguaje más elaborado.

Cabe aclarar que estas categorías, surgieron de las interrogantes realizadas a cada uno de ellos, pero como en las entrevistas habían preguntas similares y otras no, se procedió a presentarlas como categorías coincidentes y categorías no coincidentes las cuales son apoyadas por el cuadro anterior se presenta el siguiente orden de presentación definiciones de autores y en algunos casos con definiciones de los entrevistados.

\section{Categorías coincidentes}

\section{Autodefinición de emprendedor}

Para los estudiantes y egresados ellos lo definen como la persona que se cree capaz de iniciar su propia empresa bien sea grande o pequeña, de tomar sus propias decisiones, tiene ideas, conocimientos de lo quiere lograr, bueno, si llegara a montar su propio negocio debe tener la responsabilidad de ponerlo en marcha, sin importar el riesgo que se pueda presentar en el desarrollo de sus actividades o como aquella que tiene iniciativa y busca su propio destino laboral 
creyendo en sus ideales y se caracteriza por ser valiente y capaz.

Emprendimiento, es entonces la posibilidad que tienen las personas que han estudiado la necesidad de un producto, proceso, un bien para uso colectivo entre otros, para proceder a fabricarlos, a negociarlo organizándose e invirtiendo dinero el cual implica correr riesgos, esto con la finalidad de crear un beneficio en la comunidad para solucionar una necesidad en la misma.

Según Shaver y Scott (1991) al reconocer esta realidad señala:

Las circunstancias económicas son importantes, las redes son importantes; los equipos de emprendimiento son importantes, los mercados, las finanzas; e incluso la asistencia de las agencias públicas son importantes. Sin embargo, ninguno de éstos por separado es capaz de crear un nuevo emprendimiento. Para ello necesitamos una persona que contemple en su mente todas las posibilidades juntas y que crea que la innovación es posible, pero que además tenga la motivación para persistir hasta que el trabajo esté completo (p. 25).

Ahora bien, la presente afirmación expresa que el emprendimiento se deduce aquí como actitud hacia la búsqueda de decisiones estimuladas por el logro y el éxito personal; por el apego al poder y al ejercicio del control, por la tendencia que se tiene al desafío y la ambición que gobierna la toma de decisiones. El emprendedor se mueve con libertad es por ello que siempre presenta la perseverancia y la determinación como experiencias frecuentes que reflejan su confianza y entusiasmo por la novedad.

\section{Características del emprendedor}

El cambio forma parte fundamental del espíritu del emprendedor con habilidades definidas conscientes de que como parte de la sociedad y actores de evolución a partir del emprendimiento, se generaran nuevas fuentes de trabajo dirigida hacia una sociedad digna, donde exista igualdad de oportunidades para toda las personas que buscan mejor calidad de vida y así se produzcan nuevas transformaciones en la sociedad. Según Cardozo, (2010) define el emprendimiento como:

Una manera de pensar y actuar, orientada hacia la creación de riqueza, a través del aprovechamiento de oportunidades. Es el conjunto de valores, creencias, ideologías, hábitos, costumbres y normas, que comparte un grupo de personas hacia el emprendimiento, sólo los que están dispuestos a tomar decisiones están dispuestos aprender y desaprender en búsquedas de nuevas oportunidades. Oportunidades de negocios ahí donde otras personas no alcanzan a observar la generación de nuevos emprendimientos que generen nuevos ingresos para su crecimiento económico. (p. 87).

\section{Auto reconocimiento del "yo" emprendedor}

Autoreconocimiento: Es el proceso reflexivo por el cual la persona adquiere noción de su yo y de sus propias cualidades y características. Para Herrera (2009): 
El reconocerse como emprendedor es reconocer que se es una persona que habitualmente siente que está sometida a muchísimas evaluaciones: la suya propia, la de sus socios, equipo de trabajo, inversores, proveedores... y que, a la vez, evalúa al resto. Por eso me parece especialmente interesante, a la par que contribuirá a su propio desarrollo y al de su negocio, el saber dar y recibir reconocimiento.

Por su parte los estudiantes y egresados señalan que tienen características de emprendedores porque se sienten capaces de asumir toda responsabilidad que se le presente, cuidar su dinero, tener compromiso personal en el momento de que se presente la oportunidad de ocuparse en algún trabajo que me salga, de tal manera que me conduzca a lograr todo lo que quiero en mi vida. Son optimistas, responsables y sobre todo comprometidos con lo que hacen y eso es lo que importa para lograr cualquier objetivo.

\section{Origen de la actitud emprendedora}

No se sabe a ciencia cierta el origen de la actitud emprendedora, por ello según Shaver y Scott (1991) esto del emprendimiento existen muchos mitos y realidades se dice que para ser emprendedor se debe nacer con esa cualidad y no tratar de serlo en el camino, pero en realidad se puede tener inteligencia innata, llevando a convertir a las personas en emprendedores por acumulación de habilidades, conocimiento práctico, experiencia y contactos.

\section{Clave para el triunfo profesional del emprendedor y elementos que contribuyen al emprendimiento}

Para Gaviria (2012) existen muchos factores pero el que prevalece es la Motivación del Empresario: principal motivo para aprovechar la oportunidad de emprender. Se plantean diversas razones: motivación primaria de emprendimiento o deseo de poner un negocio, existencia de oportunidades, modelo de referencia o haber conocido personalmente a un emprendedor en los dos últimos años, autoempleo, expectativas de ingresos, y miedo al fracaso para emprender, es decir, si en el país o región la mayoría de la gente opina que iniciar un negocio es una opción profesional atractiva.

\section{Capacitación y emprendimiento}

Para Gaviria (2012). Educación y capacitación. Se refiere a la medida en que el conocimiento y las habilidades para crear o dirigir emprendimientos (nuevos o en crecimiento) son incorporados dentro del sistema educativo y de capacitación en todos los niveles (primaria, secundaria, técnico y universitario). Por su parte los expertos piensan que es importante pero no indispensable, si la gente se capacita y se forma para tener un conocimiento, pero si ya los tiene solo debe actualizarse constantemente sobre lo que está pasando con el producto, la competencia, los procesos, la tecnología y fundamentalmente lo que está pasando con el mercado, el mercado entendido como cliente, hay que estar atentos con los exigencias del cliente. Para otro de los expertos la capacitación es clave a la hora del emprendimiento, porque muchas personas pueden haber alcanzado un cierto nivel de éxito sin haber estudiado pero cuando tu revisa su bibliografía o la historia de su éxito te das cuenta que ellos tuvieron que enfrentar o lidiar con 
elementos que para una persona que quizás está capacitada le fue más fácil sortear

\section{Auto significado de resiliencia}

Se entiende como la capacidad del individuo para hacer las cosas bien, pese a situaciones adversas, teniendo en cuenta la firmeza y una facultad de construcción positiva de las personas, de una familia o de un grupo social de desenvolverse positivamente, a pesar de tener condiciones de vida difíciles. Resiliencia según Grotberg (1998:130), es "La capacidad del ser humano para hacer frente a las adversidades de la vida, superarlas y ser transformado positivamente por ellas". Al desarrollar esta capacidad, la persona no solo afrontará situaciones difíciles sino que además conseguirá una manera satisfactoria de sacarle provecho y progresar de forma personal y profesionalmente, logrando todo lo que se proponga.

\section{Autoreconocimiento como persona resiliente}

La resiliencia implica reestructurar nuestros recursos psicológicos en función de las nuevas circunstancias y de nuestras necesidades. De esta manera, las personas resilientes no solo son capaces de sobreponerse a las adversidades que les ha tocado vivir, sino que van un paso más allá y utilizan esas situaciones para crecer y desarrollar al máximo su potencial. De acuerdo con Garmezy, Norman. (1991) Para las personas resilientes no existe una vida dura, sino momentos difíciles. Y no se trata de una simple disquisición terminológica, sino de una manera diferente y más optimista de ver el mundo ya que son conscientes de que después de la tormenta llega la calma. De hecho, estas personas a menudo sorprenden por su buen humor $y$ nos hacen preguntarnos cómo es posible que, después de todo lo que han pasado, puedan afrontar la vida con una sonrisa en los labios.

\section{Emprendimiento como mito o realidad}

Según Shaver y Scott (1991) al reconocer esta realidad señala:

Las circunstancias económicas son
importantes, las redes son
importantes; los equipos de
emprendimiento son importantes,
los mercados, las finanzas; e incluso
la asistencia de las agencias públicas
son importantes. Sin embargo,
ninguno de éstos por separado es
capaz de crear un nuevo
emprendimiento. Para ello
necesitamos una persona que
contemple en su mente todas las
posibilidades juntas y que crea que
la innovación es posible, pero que
además tenga la motivación para
persistir hasta que el trabajo esté
completo" (p. 25).

Ahora bien, la presente afirmación expresa que el emprendimiento se deduce aquí como actitud hacia la búsqueda de decisiones estimuladas por el logro y el éxito personal; por el apego al poder y al ejercicio del control, por la tendencia que se tiene al desafío y la ambición que gobierna la toma de decisiones. El emprendedor se mueve con libertad es por ello que siempre presente la perseverancia y la determinación como experiencias frecuentes que reflejan su confianza y entusiasmo por la novedad. Lo cierto es que en esto del emprendimiento existen muchos mitos y realidades se dice que para ser emprendedor se debe nacer con esa cualidad y no tratar de serlo en el camino, pero en realidad se puede tener inteligencia innata, llevando a convertir a las personas en emprendedores por 
acumulación de habilidades, conocimiento práctico, experiencia y contactos.

Para los expertos el emprendimiento es una gran realidad, porque históricamente muchos países, han sufrido profundas depresiones en el mundo económico, ya que los procesos macro económicos entran en una profunda crisis quienes salen a relucir son los procesos micro económicos y en estos procesos él punto fundamental es el emprendimiento, un mito es un relato de un acontecimiento legendario, es por ello que emprender no es un mito sino una realidad, como les decía el ser humano tiene la habilidad para poder emprender, avanzar, desarrollarse y crecer en la vida, en su propio instinto busca la manera de salir adelante para poder cubrir sus necesidades y las de su familia.

\section{Clarificar la conducta resiliente como mito o realidad}

Córdova, Andrade y Rodríguez (2005) La resiliencia es una realidad, la presente afirmación expresa que toda experiencia negativa en alguna medida mayor o menor deja en quien la vive algún efecto que marca su vida, a veces no se observa a simple vista $y$ en otras ocasiones los efectos aparecen después de haber pasado mucho tiempo. Existen muchas definiciones de resiliencia aunque todas tienen siempre un común denominador, que no es más que la capacidad de una persona de hacer las cosas bien pese a las condiciones que haya tenido en su vida, superarlas frustraciones y salir de ellas reforzado 0 incluso renovado, recuperándose y permitiéndose tener una vida significativa y productiva, para sí y para la sociedad en la que se encuentra inserto.

Pero la resiliencia no es una condición o una cualidad innata, algo que tenemos o no tenemos en función de los caprichos del destino o de nuestra buena fortuna. Nada de eso. La resiliencia es algo que todos, sin excepción, podemos desarrollar, aunque en ocasiones para lograrlo sea necesario modificar algunas de nuestras creencias, convicciones y hábitos.

\section{Categorías no coincidentes}

\section{Limitantes del emprendimiento}

Cardozo, (2010) dice que los seres humanos están cargados de creencias que impiden y bloquean nuestras acciones, ya sea por nuestra propia experiencia o aprendizaje como por la capacidad de nuestro cerebro para crear situaciones. Estas creaciones de nuestra mente pueden estar fundamentadas en vivencias reales del mundo que le rodea, como la vida de alguien que conocemos, o bien en películas, cuentos, historias, anécdotas y cualquier elemento susceptible de alimentar nuestros miedos y temores, ideas o creencias. Por eso podemos decir que nuestra mente está entrenada en la limitación y no en la abundancia, y para poder emprender, el objetivo principal es generar valor. $Y$ no puede haber generación de valor si nosotros pensamos que no podemos influir en cualquier circunstancia por compleja que sea.

\section{Resistencia al cambio y resiliencia}

No se consiguieron autores que definieran esta diferencia, pero los expertos opinan que la resistencia al cambio no forma parte de la resiliencia ya que esta no siempre tiene que ver con bloqueos mentales de la gente, son paradigmas que manejan que pueden ser vencidos o pueden ser cambiados. Para ellos la persona resiliente es aquella que desde la adversidad se transforma y al transformarse va viendo un conjunto de habilidades y potencialidades 
que le permiten descubrir esa fuerza o poder del que se dispone para lograr un fin, Creen que resistirse al cambio es bloquearse al crecimiento y desarrollo personal, una persona resiliente puede adaptarse, busca crecer busca la manera de subsistir o desarrollarse en medio de cualquier circunstancia. La resistencia al cambio es un obstáculo para el crecimiento personal.

\section{Diferencias entre emprendimiento y resiliencia}

No se consiguieron autores que definieran esta diferencia, pero los expertos opinan que conseguir diferencias entre estos dos conceptos o definiciones es difícil porque ellas se integran, la resiliencia es producto de conductas y maneras de pensar que le dan la oportunidad a las personas progresar y desenvolverse tomando las situaciones que estén a su favor, y las que no también. Es difícil conseguir diferencias porque ellas se complementan, la resiliencia es fruto de conductas y formas de pensar que permiten a las personas crecer y desenvolverse utilizando circunstancias que estén a su favor, y no a pesar de ellas. Yo no veo mucha diferencia, la única diferencia es que la resiliencia aplica a todas las áreas de la vida, a lo familiar, personal y todo eso. El emprendimiento se enfoca más hacia el área de los negocios.

\section{Elementos favorecedores de la resiliencia \\ Para Cyrulnik, (2007) una persona con tenacidad, con capacidad para volver a iniciar algo, que en los primeros intentos no logró obtener lo esperado del proyecto que inicio, es decir, el ejemplo que siempre se pone es el de la gente que se cae y vuelve a levantarse. Indudablemente que el tema de la seguridad en sí mismo forma un papel}

importante, para ello hay que educar a la persona para que vaya viendo sus fortalezas, debilidades y desde allí pueda entender las circunstancias, debe ser una persona con una autoestima, una persona resiliente tiene que ser muy parecido al emprendedor tiene que ser perseverante, una persona de mucha fe, mucha fe en sí mismo y fe en Dios, ver más allá de su propia realidad y ser muy firme, tener constancia y perseverancia en lo que se ha propuesto.

\section{Factores obstaculizantes de la resiliencia}

Cyrulnik, (2007) cita como factores obstaculizantes la falta de confianza en sí mismo, no ser perseverante y falta de autoestima. De acuerdo con los estudiantes, estos señalaron el miedo y uno de los más importante es el económico porque lo que uno gana no le alcanza para nada, todo se va en comprar comida y no queda para poder reunir como para uno pensar en montar un negocito aunque sea en su casa para evitarse el alquiler y todo los demás gastos. Pero también piensa que la conducta de la persona, sino es capaz de controlarse esto no le permitirá continuar.

\section{Los sujetos y su visión del problema}

La determinación de las categorías a partir de la discursiva de los sujetos de estudio así como el contenido de las mismos se analizan conforme a los siguientes criterios: B.1.- Los sujetos ponen orden discursivo: Criterios y contenidos, B.2.Análisis las debilidades y fortalezas. B.3.Teorización triangular: Construcción de la visión en su conjunto. 
Los sujetos ponen Orden Discursivo: EI discurso. Criterios y contenidos (Estudiantes- Egresados- Expertos)

De acuerdo con el discurso de los estudiantes entrevistados, cuando se les pregunta que sería para ellos una persona emprendedora, ellos opinan que es la que se cree capaz de iniciar su propia empresa bien sea grande o pequeña, de tomar sus propias decisiones, tiene ideas, conocimientos de lo quiere lograr, otro no muestra mucho conocimiento sobre el significado de lo que es ser emprendedor pero piensa que está relacionado con la oportunidad que tiene una persona al momento de querer montar o abrir un negocio propio. El otro estudiante piensa que es el que crea o logra cosas nuevas por sus propios medios, y alcanza las metas que quiere sin depender de alguien más, sino de lo que conoce, habilidades que tiene. Acerca de las características que debe tener una persona emprendedora, piensan que debe ser una persona honesta, trabajadora, responsable, capaz de resolver cualquier problema que se les presente $y$ dispuesta a tomar las decisiones que le beneficien, una persona con ideas, agilidad para los negocios, responsabilidad, compromiso y sobre todo tener dinero para poder realizar ese proyecto, también mencionan la responsabilidad, tener una visión de futuro, también debe ser capaz de tomar riesgos, de tener confianza en sí mismo.

En cuanto a auto reconocerse como persona emprendedora expresan que si se consideran emprendedores porque son capaces de asumir toda responsabilidad que se le presente, no obstante, colocan como limitante la situación del país, al no haber oportunidades ni dinero. Otro de los estudiantes expresó que gracias a los conocimientos que se puedan adquirir, se puede buscar la manera de no depender de un patrón, si no ser el dueño de su propio negocio, y dueño de su tiempo y dinero, dando preponderancia al estudio como factor clave. Para ellos el emprendedor es un ser que aprende a lo largo de la vida desde que nace, hasta que se muere, es decir que siempre está interesado en conocer cosas nuevas, saber qué hacer, para aplicarlo en la vida. Para otros un emprendedor nace o se hace debido a que ellas son personas que son optimistas y siguen creyendo en un porvenir.

Con respecto a saber si para usted poder tener su propio negocio le haría falta mucho dinero o por el contrario lo importante serían sus habilidades, para los estudiantes hace falta las dos cosas porque se trata de iniciar algo nuevo y siempre que se va comenzar se necesita dinero, tiempo, esfuerzo y dedicación, otro estudiante expresa que en estos momentos y con la situación del país es importantísimo e indispensable el dinero, ya que por muchas habilidades y todas las ideas que pueda tener una persona con ánimos de montar su propio negocio para independizarse sin dinero nada se hace. Por tanto se necesitaría de las dos cosas, para poder soportar y mantenerse, porque no solamente necesitamos el talento, sin dinero es muy difícil realizar algún trabajo. Ellos opinaron que la capacitación laboral adquirida en la Asociación Civil Juventud y Trabajo es una oportunidad para usted emprender su propio negocio ya que les permite desarrollar nuevos conocimientos, y prepararse para asumir las pruebas que se le pongan en el camino, obtener más experiencia para poder iniciar su propio negocio. Los conocimientos que se adquieren en la asociación son muy buenos, pero a veces es lamentable y desmotivador 
estudiar con la esperanza de que al finalizar tus estudios los conocimientos adquiridos se puedan poner en práctica, lo cual no todas las veces ocurre, pero si consideran necesario aprender un oficio.

Los estudiantes expresaron no conocer a fondo lo que es la resiliencia, pero tienen una noción de que se trata de cuando una persona es capaz de aceptar la realidad tal y como es; y que así haya tiempos difíciles como los que estamos pasando se puede salir adelante. Es la fuerza que deberían tener todos de recuperarse de un fracaso ante tantos problemas y tantas dificultades para así intentar sobrevivir. No están muy claros en el significado pero creen que tiene que ver con salir adelante en una situación difícil. Ellos creen que son resilientes al ser capaces de enfrentar nuevos retos que le permitirán salir adelante conseguir lo que quieren, mejorar su calidad de vida, ser independiente y de montar su propio negocio y con esta situación que afecta a
Venezuela piensan que todas las personas deben asumir nuevos desafíos, ya que es indispensable ser optimista y comenzar de nuevo, más aun si hay una familia que mantener y que cuidar, se debe hacer todo lo que se pueda para salir adelante; claro todo lo que sea correcto. Personalmente creen que con su esfuerzo y motivación pueden salir adelante luego de un fracaso, a pesar de la situación que actualmente se vive en el país, hay que ser positivo y cuidar todo lo que tiene, porque aunque estemos pasando por esta situación debemos siempre sacar lo bueno de cada problema.

\section{Tejiendo una red de relaciones}

De acuerdo al cuadro de categorías extraídas de las entrevistas realizadas, se procedió a sintetizar para determinar e identificar las palabras clave que están relacionadas con una persona emprendedora en la siguiente figura:

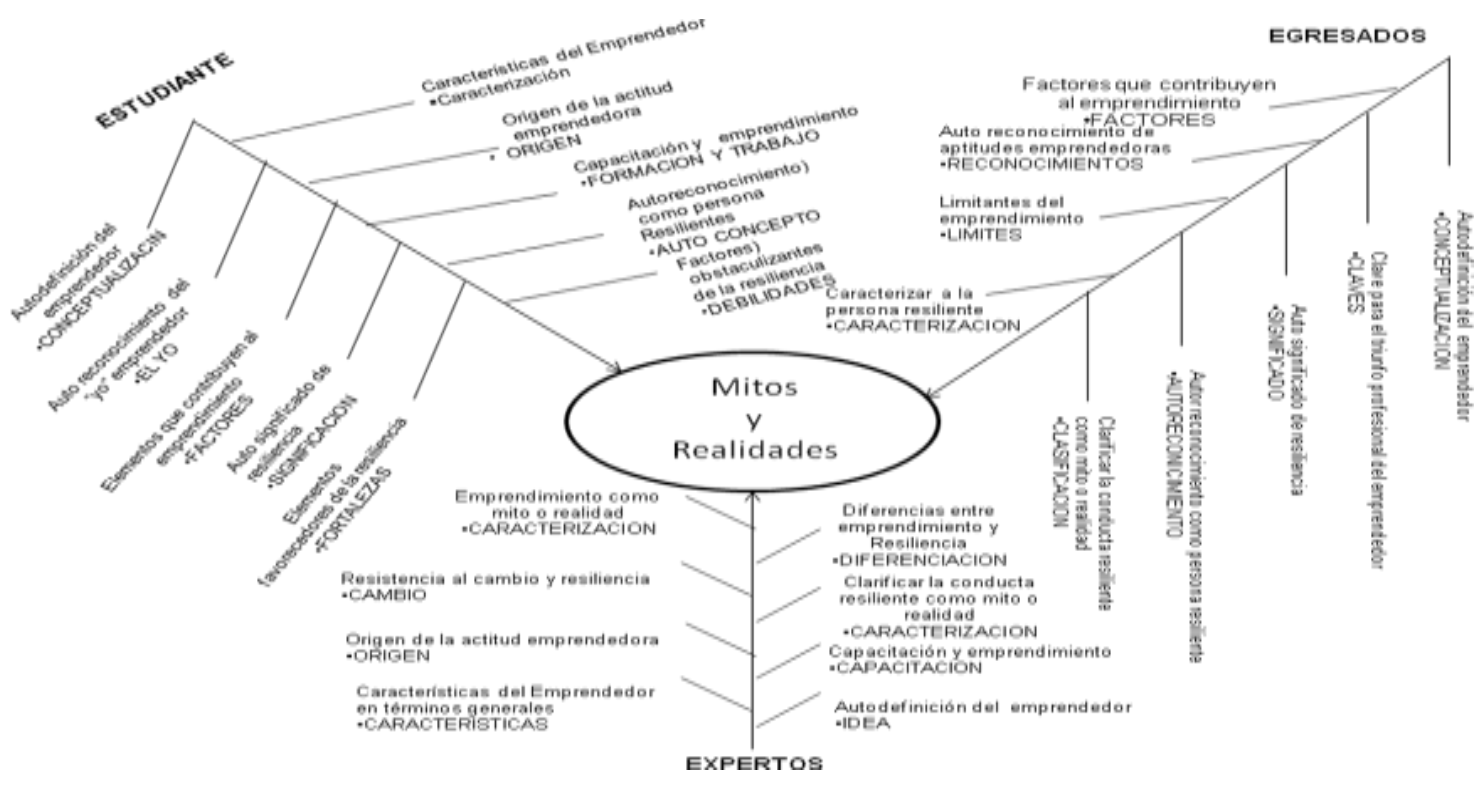

Figura 1. Categorías de los informantes claves con relación emprendimiento 


\section{CONCLUSIONES}

Se puede reflexionar que al analizar el emprendimiento y la resiliencia desde la perspectiva de los mitos y realidades en una Entidad no gubernamental en el área del emprendimiento, es ahondar en estos grandes temas, por demás muy interesantes y de relevancia contemporánea, a través de la visión de tres grupos (estudiantes, egresados y expertos) determinando su posición con respecto al tema, así como su conocimiento para definirlos, reconocer las características de su yo emprendedor o resiliente, valorar las características de los sujetos en relación a su situación de emprendedor y entre los aspectos más importantes reconocer el efecto de la capacitación en el emprendimiento. Cabe destacar, que este trabajo investigativo fue producto de un arduo trabajo, donde las investigadoras desde el método etnográfico se apropiaron de una realidad para conocerla, reflexionar sobre ella y extraer sus propias conclusiones en función de los hallazgos encontrados.

\section{REFERENCIAS}

Cardozo, A. (2010). La motivación para emprender. Evolución del modelo del rol en emprendedores argentinos [Tesis Doctoral]. Universidad Nacional de Educación a Distancia, Facultad de Ciencias Económicas y Empresariales, Departamento de Organización de Empresas. Recuperado de http://goo.gl/ Y98AIZ. Fecha de consulta 24 ene 017

Córdova-Alcaráz, Alberto Javier; Andrade Palos, Patricia; Rodríguez-Kuri, Solveig Eréndira (2005). Características de resiliencia en jóvenes usuarios y no usuarios de drogas. En Revista Intercontinental de Psicología y
Educación vol. 7, núm. 2, juliodiciembre, pp. 101-122

Cyrulnik, B. (2007). Los patitos feos. La resiliencia: una infancia infeliz no determina la vida. Gedisa

Dahms, T. (2010), "Resilience and risk manage-ment", The Australian Journal of Emergency Management, vol. 25, núm. 2, abril, pp. 6-21

Garmezy, N. (1991). Resilience in children's adaptation to negative life events and stressed environments. PediatricAnnal, (pp.459- 466).Cambridge, England: Cambridge University Press

Gaviria Nieto, L. M. (2012). Optimizar la comunicación interna en COODESURIS, mediante la implementación de estrategias que fortalezcan la identidad corporativa.

Grotberg, E. (1998), "Manual de identificación de la resiliencia en los niños y adolescentes". Organización Panamericana de la Salud

Herrera, H. (2009). Investigación sobre redes sociales y emprendimiento: revisión de la literatura y agenda futura. Disponible en

http://www.revistas.unal.edu.co/index. php/innovar/article/view/20959/36361. Fecha de consulta: 24 ene 2017

López, A. (2009), "El lado positivo de la crisis", CNNexpansión.com, 28 de octubre, en <www. cnnexpansion.com/manufactura/2009/1 0/28/el-lado-positivo-de-la-crisis> [consultado el 18 de abril de 2011]

Melillo, A., Y Suárez Ojeda, E. (2001). Resiliencia. Descubriendo las propias fortalezas. Buenos Aires: Paidós

Schumpeter, Joseph (1994). Publicado por Routled-ge. USA

Shaver, K. y Scott, L. (1991). Person, process, choice: The psychology of new venture 
creation, Entrepreneurship: Theory and Practice, na 16, vol 2, pp. 23-45.

Smith, A. y F. Graetz (2011), Philosophies of Organizational Change, Reino Unido, Edward Elgar

Werner, E. E. (2005). Resilience research. In Resilience in children, families, and communities (pp. 3-11). Springer, Boston, MA.

Torres, L. (2014). Sistema Lerma: una visión política en la gestión pública del agua, ¿solución Estatal o Federal? ISBN: 978607-8087-16-7

Westrum, R. (2006) "A Typology of Resilience Situations" en E. Hollnagel, D. Wooods y $N$. leveson, Resilience engineering: concepts and precepts, reino Unido, ashgate 